\title{
Frozen in darkness
}

In 2014, water vapour was detected around Ceres, a dwarf planet in the asteroid belt. NASA's Dawn spacecraft, in orbit around Ceres since March 2015, subsequently found water ice on the dwarf planet, in a small, mid-latitude crater named Oxo. Now, writing in Nature Astronomy, Platz et al. show that polar regions of Ceres that lie in perpetual darkness trap and preserve water ice, providing valuable information about the processes that create water reservoirs on planetary bodies (T. Platz et al. Nature Astron. http://dx.doi.org/10.1038/s41550-0160007; 2016).

Permanently shadowed areas on airless planetary bodies, usually found in craters at polar latitudes, have favourable conditions for retaining water ice. Without sunshine, these regions are extremely cold (a few tens of kelvin), and can therefore efficiently trap water molecules and retain them for geological timescales. This cold-trapping process is known to be active on the Moon and on Mercury.

Platz and colleagues analysed images obtained by Dawn's Framing Camera to reconstruct complete maps of the shadowed areas of the northern polar region of Ceres (pictured). They find more than 600 craters in perennial shadow, 10 of which exhibit bright features. One of these features has a (partially) illuminated portion, allowing Platz et al. to perform a spectral analysis using Dawn's imaging spectrometer. The spectra show clear signatures of water ice, providing definitive evidence for the nature of these bright deposits.

The authors also constrain the timescale

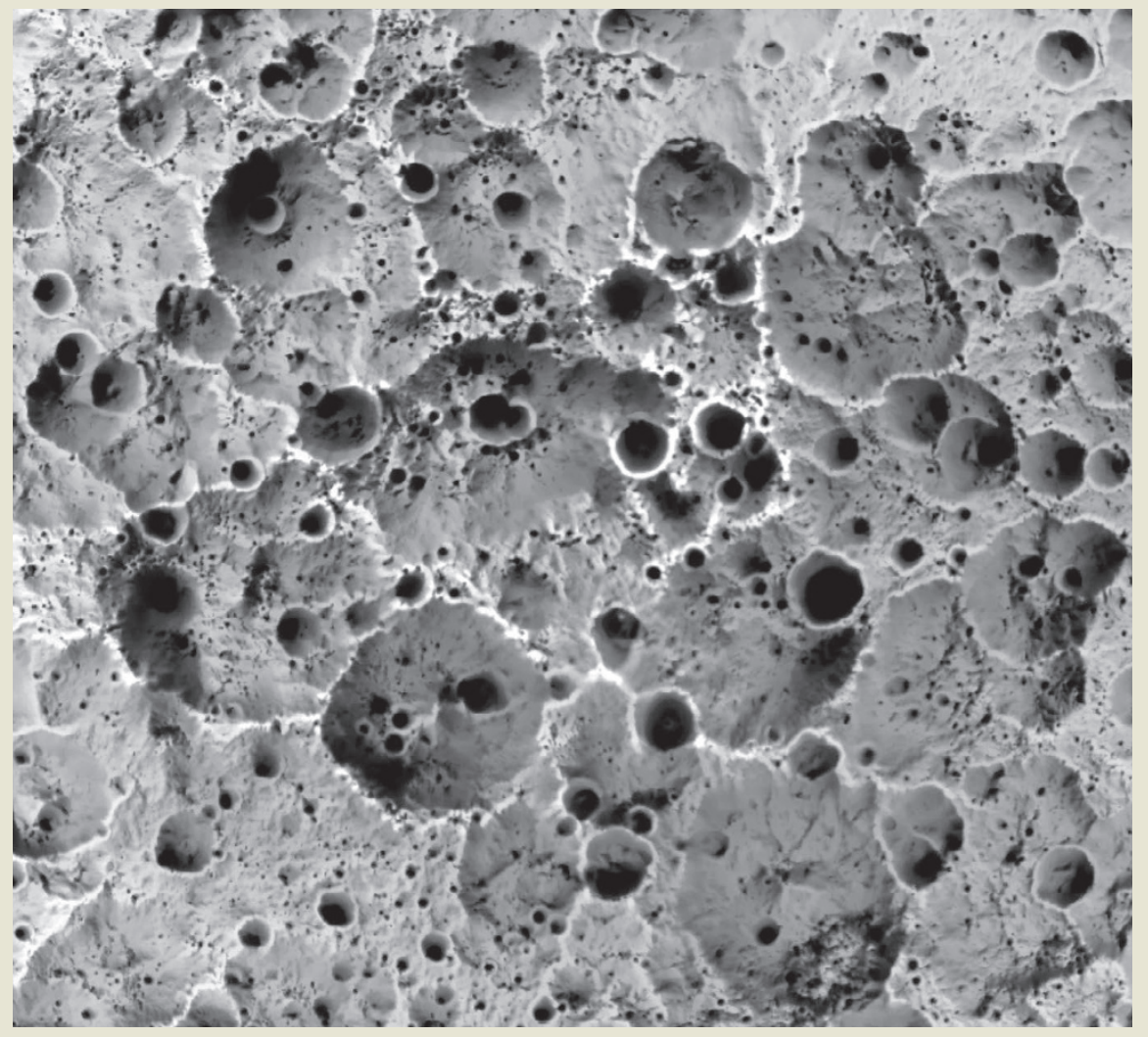

of water-ice production on Ceres using the fact that ice deposits evolve through two competing mechanisms: slow accumulation by cold-trapping and destruction by impact 'gardening', the overturning of planetary soil by impacts. The authors estimate that the deposits are young (not more than a few hundred thousand years old), which implies that ice delivery is continuously active. In addition, the deposits are relatively scarce (compared with, for example, those on Mercury), suggesting that Ceres is unable to retain much water or didn't have much to begin with. Luca Maltagliati

This article was published online on 19 December 2016.

\section{Quantum mechanics in a spin}

Quantum spin liquids are exotic states of matter first predicted more than 40 years ago. An inorganic material has properties consistent with these predictions, revealing details about the nature of quantum matter. SEE LETTER P.559

\section{LEON BALENTS}

$\mathrm{T}$ he phenomenon of magnetism, discovered thousands of years ago, arises from the alignment of electron magnetic moments known as spins. But if these spins do not align, they can form a truly quantum state called a quantum spin liquid (QSL). On page 559, Shen et al. ${ }^{1}$ report measurements of exotic spin excitations in an inorganic material (of ytterbium, magnesium, gallium and oxygen; $\mathrm{YbMgGaO}_{4}$ ). The authors' observations suggest that $\mathrm{YbMgGaO}_{4}$ forms a QSL that is closely analogous to a state of matter associated with electrons in a metal. The appearance of electron-like particles in such a material is surprising, and indicates extraordinary 'quantum entanglement' of the underlying spins.

The concept of a QSL was first introduced ${ }^{2}$ in 1973 by the physicist Philip Anderson, who described such a system as "resonating", indicating the presence of quantum superposition - in quantum mechanics, reality is represented by a wavefunction in which physical states of a system can be added together like numbers in arithmetic. An extreme example of quantum superposition is Schrödinger's famous cat, whose wavefunction is the sum of the state of the living cat and that of the dead cat. The QSL is a close relative of Schrödinger's cat that incorporates long-range entanglement (a superposition involving many widely separated spins). According to modern quantum theory, this type of entanglement is so stable 